University of Warwick institutional repository: http://go.warwick.ac.uk/wrap This paper is made available online in accordance with publisher policies. Please scroll down to view the document itself. Please refer to the repository record for this item and our policy information available from the repository home page for further information.

To see the final version of this paper please visit the publisher's website. Access to the published version may require a subscription.

Author(s): T. D. Veal, L. F. J. Piper, S. Jollands, B. R. Bennett, P. H. Jefferson, P. A. Thomas, and C. F. McConville, B. N. Murdin, L. Buckle, G. W. Smith, and T. Ashley

Article Title: Band gap reduction in GaNSb alloys due to the anion mismatch

Year of publication: 2005

Link to published version: http://dx.doi.org/10.1063/1.2058224

Publisher statement: None 


\title{
Band gap reduction in GaNSb alloys due to the anion mismatch
}

\author{
T. D. Veal, L. F. J. Piper, S. Jollands, B. R. Bennett, ${ }^{a)}$ P. H. Jefferson, \\ P. A. Thomas, and C. F. McConville \\ Department of Physics, University of Warwick, Coventry, CV4 7AL, United Kingdom \\ B. N. Murdin \\ School of Electronics and Physical Sciences, University of Surrey, Guildford, GU2 5XH, United Kingdom \\ L. Buckle, G. W. Smith, and T. Ashley \\ QinetiQ Ltd., St. Andrews Road, Malvern, Worcestershire, WR14 3PS, United Kingdom
}

(Received 25 March 2005; accepted 3 August 2005; published online 19 September 2005)

\begin{abstract}
The structural and optoelectronic properties in $\mathrm{GaN}_{x} \mathrm{Sb}_{1-x}$ alloys $(0 \leqslant x<0.02)$ grown by molecular-beam epitaxy on both GaSb substrates and AlSb buffer layers on GaAs substrates are investigated. High-resolution x-ray diffraction (XRD) and reciprocal space mapping indicate that the $\mathrm{GaN}_{x} \mathrm{Sb}_{1-x}$ epilayers are of high crystalline quality and the alloy composition is found to be independent of substrate, for identical growth conditions. The band gap of the GaNSb alloys is found to decrease with increasing nitrogen content from absorption spectroscopy. Strain-induced band-gap shifts, Moss-Burstein effects, and band renormalization were ruled out by XRD and Hall measurements. The band-gap reduction is solely due to the substitution of dilute amounts of highly electronegative nitrogen for antimony, and is greater than observed in GaNAs with the same $\mathrm{N}$ content. () 2005 American Institute of Physics. [DOI: 10.1063/1.2058224]
\end{abstract}

Dilute nitride arsenides, such as $\mathrm{GaN}_{x} \mathrm{As}_{1-x}$ and $\mathrm{Ga}_{1-y} \mathrm{In}_{y} \mathrm{~N}_{x} \mathrm{As}_{1-x}$, have been demonstrated to have considerable potential for optoelectronic applications. The substitution of nitrogen on a few percent of the arsenic sites reduces the band gap and lattice constant simultaneously, allowing GaInNAs alloys with band gaps in the optical fiber wavelength range of 1.3 to $1.55 \mu \mathrm{m}$ to be lattice matched to GaAs. ${ }^{1}$ While dilute nitride arsenides have been heavily studied, dilute nitride antimonides have received relatively little attention. For instance, a recent review by Vurgaftman and Meyer of nitrogen containing semiconductors highlighted a complete lack of experimental results on $\mathrm{GaN}_{x} \mathrm{Sb}_{1-x},{ }^{2}$ even though band-gap reduction has been observed in $\mathrm{InN}_{x} \mathrm{Sb}_{1-x}$. ${ }^{3,4}$ Although the growth of $\mathrm{GaN}_{x} \mathrm{Sb}_{1-x}$ as a step towards realizing the growth of Sb-rich GaInNAsSb has recently been reported, ${ }^{5}$ no optical studies of the $\mathrm{GaN}_{x} \mathrm{Sb}_{1-x}$ alloy were presented.

In dilute nitrides, the substitution of highly electronegative nitrogen for a few percent of the host anions results in the formation of a highly localized resonant band close to the conduction band minimum. ${ }^{6,7}$ The band-gap reduction due to the localized interaction between the host conduction band and the narrow resonant level, formed by the nitrogen states, can be described by the band anticrossing (BAC) model. ${ }^{6}$ Considering that the electronegativity mismatch between nitrogen and antimony is greater than for any other combination of commonly used group $\mathrm{V}$ elements, the band-gap reduction in dilute nitride antimonides is expected to be more extreme than for dilute nitride arsenides. Furthermore, dilute nitride antimonides have useful potential device applications. For instance, the $\mathrm{GaN}_{x} \mathrm{Sb}_{1-x}$ ternary alloy is suitable for $2-5 \mu \mathrm{m}$ midinfrared applications and the related quaternary,

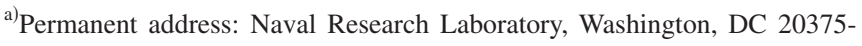
5347.

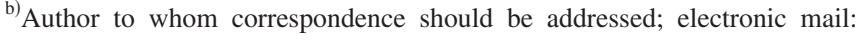
C.F.McConville@warwick.ac.uk
}

$\mathrm{Ga}_{1-y} \mathrm{In}_{y} \mathrm{~N}_{x} \mathrm{Sb}_{1-x}$, can be lattice matched to GaSb.

Here, the structural, optical, and transport properties of dilute $\mathrm{GaN}_{x} \mathrm{Sb}_{1-x}(0 \leqslant x<0.02)$ thin films are reported. The $\mathrm{GaN}_{x} \mathrm{Sb}_{1-x}$ samples were grown using nitrogen plasmaassisted molecular-beam epitaxy (MBE); details can be found elsewhere. ${ }^{8}$ Thin films (nominally $250 \mathrm{~nm}$ ) of $\mathrm{GaN}_{x} \mathrm{Sb}_{1-x}$ were grown on undoped $\mathrm{GaSb}(001)$ substrates with 150-nm GaSb buffer layers. Thicker $\mathrm{GaN}_{x} \mathrm{Sb}_{1-x}$ layers $(2 \mu \mathrm{m})$ for characterization by Hall measurements and absorption spectroscopy were also grown on semi-insulating (SI) GaAs(001) substrates using a 1- $\mu \mathrm{m}$ AlSb buffer. The use of an insulating substrate, which also has a much larger band gap than the $\mathrm{GaN}_{x} \mathrm{Sb}_{1-x}$ alloys, allows the electrical and optical properties to be investigated without being affected by substrate conduction or optical absorption in the wavelength range of interest. All samples were characterized by high-resolution $\mathrm{x}$-ray diffraction (XRD) of the symmetric 004 reflection using a Philips diffractometer with $\mathrm{Cu} K \alpha$ radiation $(\lambda=0.15406 \mathrm{~nm})$. Additionally, reciprocal-space mapping around the symmetric 004 and asymmetric 224 reflections was performed on some samples using a PANalytical x-ray diffractometer. Optical absorption measurements were taken using a Perkin Elmer Spectrum GX Fourier transform infrared spectrometer, using a $\mathrm{HgCdTe}$ detector, allowing an energy range of $100-970 \mathrm{meV}$ to be investigated.

The XRD results from a GaNSb film grown on $\mathrm{GaSb}(001)$ at a temperature of $400{ }^{\circ} \mathrm{C}$ using a nitrogen plasma power of $200 \mathrm{~W}$ and with nominal dilute nitride film thicknesses of $250 \mathrm{~nm}$ are shown in Fig. 1. In addition to the substrate and epilayer peaks, a series of Pendellosung interference fringes is visible. The presence of these fringes, as well as an epilayer peak full width at half maximum of 65 arc sec close to the theoretical value (60 arc sec), indicates the layer is pseudomorphic with excellent crystalline quality. By comparison with simulated spectra, assuming Vegard's law with no lattice relaxation, a $\mathrm{N}$ fraction of $0.80 \pm 0.03 \%$ was determined for this sample. Vegard's law has been 


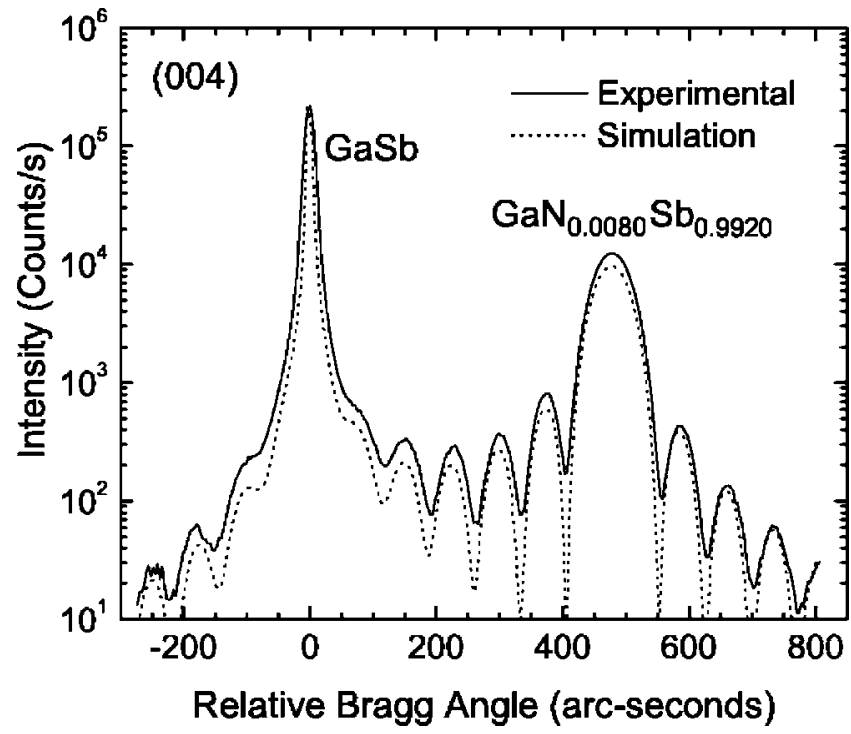

FIG. 1. X-ray diffraction (solid line) of the 004 diffraction maximum from a nominally $250-\mathrm{nm}$ GaNSb epilayer grown on a GaSb substrate at $400{ }^{\circ} \mathrm{C}$ using a nitrogen plasma power of $200 \mathrm{~W}$. The simulation (dotted lines) indicates that the anionsublattice of the GaNSb layer contains $0.80 \% \mathrm{~N}$ and that the film thickness is $252 \pm 10 \mathrm{~nm}$. The full width at half maxima of the $\mathrm{GaSb}$ and the GaNSb peaks are 16 and 65 arc sec.

shown to be valid for dilute nitrides, both theoretically ${ }^{9}$ and experimentally for $\mathrm{N}$ contents up to at least $10 \% .^{10}$

The determination of the amount of nitrogen incorporation in the $\mathrm{GaN}_{x} \mathrm{Sb}_{1-x}$ samples grown on GaAs substrates required more investigation. Since the 2 - $\mu$ m-thick $\mathrm{GaN}_{x} \mathrm{Sb}_{1-x}$ epilayers are expected to be at least partially relaxed, measurement of the symmetric 004 rocking curve is insufficient to determine uniquely the $\mathrm{N}$ content of each layer. Normally, asymmetric rocking curves, such as 224, are also measured and simulated to enable the effects of lattice relaxation and composition to be separated. However, if the epilayer is subject to tetragonal distortion, the asymmetric plane of the epilayer may become inclined with respect to that of the substrate. This means a conventional onedimensional $\omega-2 \theta$ scan is inaccurate because such a scan only measures along the [224] direction of the substrate, buffer layer, or $\mathrm{GaN}_{x} \mathrm{Sb}_{1-x}$ layer. ${ }^{11}$ Therefore twodimensional (2D) reciprocal space mapping of the 004 and 224 Bragg peaks has been performed.

The 224 reciprocal space map of a 2 - $\mu$ m-thick $\mathrm{GaN}_{x} \mathrm{Sb}_{1-x}$ epilayer is shown in Fig. 2. This sample was grown using the same temperature and $\mathrm{N}$ plasma conditions as the sample grown on GaSb shown in Fig. 1. Reciprocal space mapping revealed that the sample was almost fully relaxed and that the nitrogen content was $0.78 \pm 0.03 \%$, in agreement with the epilayer grown on a GaSb substrate $(0.80 \%)$. Additionally, these data reveal that the (224) planes of the epilayer and buffer are inclined by $0.05^{\circ}$ and $-0.10^{\circ}$, respectively, with respect to the (224) planes of the substrate. This corresponds to the residual strain in both the buffer layer and epilayer of $1.6 \pm 0.4 \%$. These results confirm that the nitrogen incorporation is independent of the substrate used, for samples grown under the same conditions. The surface morphology of samples grown on AlSb/GaAs-SI is similar to that of films grown on GaSb under the same conditions, with root mean square roughness values of typically $1.5 \mathrm{~nm}$ over a $5 \times 5 \mu \mathrm{m}$ atomic force microscopy (AFM) image. ${ }^{8}$ \[ 720 \pm 10 \mathrm{meV} \text {, very close to the value obtained from optical } \]
Downloaded 06 Jul 2009 to 137.205.202.8. Redistribution subject to AlP license or copyright; see http://apl.aip.org/apl/copyright.jsp

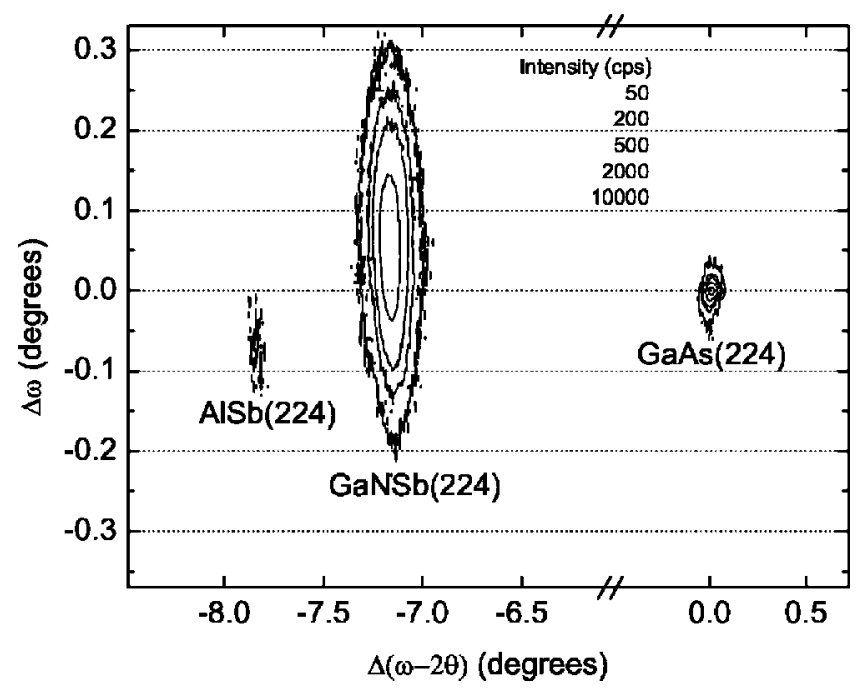

FIG. 2. Reciprocal space map of the 224 diffraction maxima from a $2-\mu \mathrm{m}$ GaNSb epilayer grown on a 1- $\mu \mathrm{m}$ AlSb buffer layer on a GaAs substrate. This sample was grown at a temperature of $400{ }^{\circ} \mathrm{C}$ using a nitrogen plasma power of $200 \mathrm{~W}$. Analysis of the 004 and 224 maps indicates that the anion sublattice of the GaNSb layer contains $0.78 \% \mathrm{~N}$ and that the epilayer is fully relaxed. The stated intensity values correspond to the contour lines in the map.

Single-field Hall measurements showed the $\mathrm{GaN}_{x} \mathrm{Sb}_{1-x}$ layers grown on $\mathrm{AlSb} / \mathrm{GaAs}$ to be $p$-type with carrier densities in the high $10^{17} \mathrm{~cm}^{-3}$ range. This $p$-type conductivity is consistent with Walukiewicz's amphoteric native defect model. ${ }^{12}$ The formation of acceptorlike impurities and defects are favored because of the proximity of the $\Gamma$-point valence band maximum (VBM) in GaSb to the universal branch-point energy, $E_{B}{ }^{13}$ The incorporation of dilute amounts of nitrogen should not change this. While the interaction in dilute nitrides between the host conduction band and the resonant nitrogen level is highly localized in $k$-space, $E_{B}$ defines the average midgap position across the entire Brillouin zone. Since the valence bands are unaffected by dilute amounts of nitrogen, ${ }^{6}$ the dilute nitride alloy $\mathrm{GaN}_{x} \mathrm{Sb}_{1-x}$ exhibits unintentional $p$-type conductivity like GaSb as a result of the Fermi level stabilizing close to $E_{B} \cdot{ }^{14}$ In GaSb, due to the lack of a sufficient density of amphoteric defects to stabilize the Fermi level, the unintentional $p$-type doping is lower than in GaNSb. In bulk- and MBE-grown GaSb, hole densities of $p \sim 10^{17} \mathrm{~cm}^{-3}$ and $p \sim$ low- $10^{16} \mathrm{~cm}^{-3}$, respectively, are typically observed. The defects responsible for the greater $p$-type conductivity in the GaNSb may be dislocations from the lattice-mismatched growth and/or damageinduced point defects created by the $\mathrm{N}$ plasma during growth.

Optical absorption spectra recorded from 2- $\mu$ m-thick $\mathrm{GaN}_{x} \mathrm{Sb}_{1-x}$ layers with different nitrogen contents $(0 \leqslant x$ $<0.02$ ) and a GaSb reference epilayer are shown in Fig. 3. The oscillations in the absorption spectra are Fabry-Perot oscillations caused by the reflection of light at the surface and at the interfaces between different materials in the samples. The turn on of the fundamental absorption obeys a square law, consistent with direct band-gap absorption. The band gap of each $\mathrm{GaN}_{x} \mathrm{Sb}_{1-x}$ epilayer is estimated by extrapolating the linear part of $\alpha^{2} E^{2}$ to the base line, where $\alpha$ is the absorption coefficient and $E$ is the photon energy. Using this method, the band gap obtained for the GaSb thin film is 


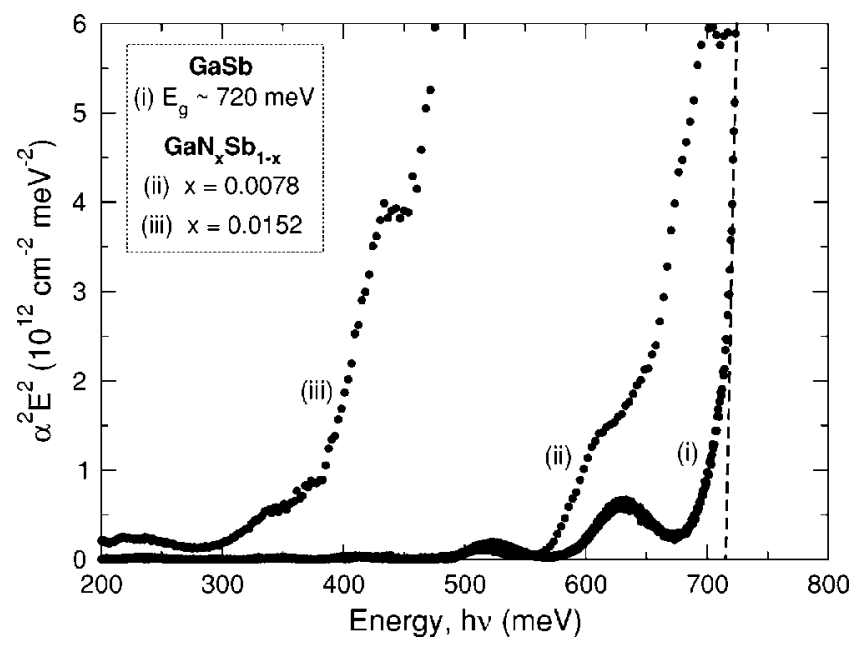

FIG. 3. Optical absorption spectra plotted as the square of $(\alpha E)$ for $\mathrm{GaN}_{x} \mathrm{Sb}_{1-x}$ layers with various compositions as a function of photon energy, $E$, recorded at $295 \mathrm{~K}$. The data shown are for (i) a $1.5-\mu \mathrm{m} \mathrm{GaSb}$ epilayer grown on $\mathrm{GaAs}$ and for $2.0-\mu \mathrm{m} \mathrm{GaN}_{x} \mathrm{Sb}_{1-x}$ epilayers grown on $\mathrm{AlSb} / \mathrm{GaAs}-\mathrm{SI}$ with (ii) $x=0.0078$ and (iii) $x=0.0152$.

absorption of 3-6 $\mu \mathrm{m}$ GaSb epilayers by Ghezzi et al. ${ }^{14}$ For the $\mathrm{GaN}_{x} \mathrm{Sb}_{1-x}$ epilayers, the absorption edge can be seen to shift towards lower energies as the nitrogen content increases. Incorporating $1.52 \% \mathrm{~N}$ into GaSb results in a band reduction of $\sim 320 \mathrm{meV}$. For comparison, the incorporation of $1.5 \% \mathrm{~N}$ in GaAs results in a band-gap reduction of $\sim 220 \mathrm{meV}^{7}$ As mentioned previously, the larger electronegativity mismatch between nitrogen and antimony (compared to nitrogen and arsenic) results in greater band-gap reduction for the same amount of nitrogen substitution.

It is known that lattice-mismatch-induced strain results in the modification of the band structure. ${ }^{15}$ Since the samples investigated here have been shown to be almost fully relaxed, for our optical studies this effect can be neglected. Additionally, effects such as the Moss-Burstein shift of the absorption edge ${ }^{16,17}$ and band-gap shrinkage due to band renormalization, ${ }^{18}$ to which narrow gap and heavily doped semiconductors can be susceptible, are negligible here. This is because the $\mathrm{GaN}_{x} \mathrm{Sb}_{1-x}$ layers are moderately $p$-type; for the measured hole densities, the bulk Fermi level lies close to the VBM. Therefore, the band-gap reduction observed can be solely attributed to the interaction between the host conduction band and the nitrogen resonant states. Further studies are required to determine the parameters of the BAC model and the role of the nonparabolic host conduction band.

To conclude, high quality $\mathrm{GaN}_{x} \mathrm{Sb}_{1-x}$ samples have been investigated by a combination of XRD, Hall measurements, and absorption spectroscopy. Full reciprocal space mapping revealed that the amount of nitrogen incorporation was independent of the substrate used, with the samples grown on GaAs substrates with AlSb buffer layers being almost fully relaxed. The optical measurements revealed a band-gap redshift with increasing nitrogen content. XRD and Hall measurements rule out contributions from strain, Moss-Burstein shift, and band renormalization. Therefore, the effect is solely due to the substitution of nitrogen for antimony. Finally, the band-gap reduction as a function of $\mathrm{N}$ content is greater than that observed in GaNAs.

The authors would like to thank Dr. David Lee and Dr. Neil Wilson for performing the Hall measurements and AFM, respectively. The Engineering and Physical Sciences Research Council, UK is acknowledged for financial support under Grants No. GR/R82630/01 and GR/S56030/01.

${ }^{1}$ M. Kondow, K. Uomi, A. Niwa, T. Kitatani, S. Watakiki, and Y. Yazawa, Jpn. J. Appl. Phys., Part 1 35, 1273 (1996).

${ }^{2}$ I. Vurgaftman and J. R. Meyer, J. Appl. Phys. 94, 3675 (2003).

${ }^{3}$ T. Ashley, T. M. Burke, G. J. Pryce, A. R. Adams, A. Andreev, B. N. Murdin, E. P. O'Reilly, and C. R. Pidgeon, Solid-State Electron. 47, 387 (2003).

${ }^{4}$ T. D. Veal, I. Mahboob, and C. F. McConville, Phys. Rev. Lett. 92, 136801 (2004).

${ }^{5}$ W. Li, J. B. Heroux, and W. I. Wang, J. Appl. Phys. 94, 4248 (2003).

${ }^{6}$ W. Shan, W. Walukiewicz, J. W. Ager, E. E. Haller, J. F. Geisz, D. J. Friedman, J. M. Olson, and S. R. Kurtz, Phys. Rev. Lett. 82, 1221 (1999).

${ }^{7}$ J. Wu, W. Shan, and W. Walukiewicz, Semicond. Sci. Technol. 17, 860 (2002).

${ }^{8}$ L. Buckle, B. R. Bennett, S. Jollands, T. D. Veal, N. R. Wilson, B. N. Murdin, C. F. McConville, and T. Ashley, J. Cryst. Growth 278, 188 (2005).

${ }^{9}$ J. Neugebauer and C. G. Van de Walle, Phys. Rev. B 51, 10568 (1995).

${ }^{10}$ T. D. Veal, I. Mahboob, L. F. J. Piper, C. F. McConville, and M. Hopkinson, Appl. Phys. Lett. 85, 1550 (2004).

${ }^{11}$ K. Uesugi, N. Morooka, and I. Suemune, Appl. Phys. Lett. 74, 1254 (1999).

${ }^{12}$ W. Walukiewicz, Appl. Phys. Lett. 54, 2094 (1989).

${ }^{13}$ J. Tersoff, Phys. Rev. B 32, 6968 (1985).

${ }^{14}$ C. Ghezzi, R. Magnanini, A. Parisini, B. Rotelli, L. Tarricone, A. Bosacchi, and S. Franchi, Phys. Rev. B 52, 1463 (1995).

${ }^{15}$ H. Asai and K. Oe, J. Appl. Phys. 54, 2052 (1983).

${ }^{16}$ T. S. Moss, Proc. Phys. Soc. London, Sect. B 67, 775 (1954).

${ }^{17}$ E. Burstein, Phys. Rev. 93, 632 (1954).

${ }^{18}$ K. F. Berggren and B. E. Sernelius, Phys. Rev. B 24, 1971 (1981). 